\title{
A Novel Segmentation Method for Furnace Flame Using Adaptive Color Model and Hybrid-Coded HLO
}

\author{
Pinggai Zhang, ${ }^{1}$ Minrui Fei $\mathbb{D}^{1},{ }^{1}$ Ling Wang $\mathbb{D},{ }^{1}$ Xian $W u,{ }^{1}$ Chen Peng, ${ }^{1}$ and Kai Chen ${ }^{1,2}$ \\ ${ }^{1}$ Shanghai Key Laboratory of Power Station Automation Technology, School of Mechatronic Engineering and Automation, \\ Shanghai University, Shanghai 200072, China \\ ${ }^{2}$ Shanghai Automation Instrumentation Co., Ltd., Shanghai 200072, China \\ Correspondence should be addressed to Minrui Fei; mrfei@staff.shu.edu.cn and Ling Wang; wangling@shu.edu.cn
}

Received 17 July 2020; Revised 14 October 2020; Accepted 31 May 2021; Published 10 June 2021

Academic Editor: Sergio Gómez

Copyright ( 2021 Pinggai Zhang et al. This is an open access article distributed under the Creative Commons Attribution License, which permits unrestricted use, distribution, and reproduction in any medium, provided the original work is properly cited.

\begin{abstract}
In recent years, the combustion furnace has been widely applied in many different fields of industrial technology, and the accurate detection of combustion states can effectively help operators adjust combustion strategies to improve combustion utilization and ensure safe operation. However, the combustion states inside the industrial furnace change according to the production needs, which further challenges the optimal set of model parameters. To effectively segment the flame pixels, a novel segmentation method for furnace flame using adaptive color model and hybrid-coded human learning optimization (AHcHLO) is proposed. A new adaptive color model with mixed variables (NACMM) is designed for adapting to different combustion states, and the AHcHLO is developed to search for the optimal parameters of NACMM. Then, the best NACMM with optimal parameters is adopted to segment the combustion flame image more precisely and effectively. Finally, the experiment results show that the developed AHcHLO obtains the best-known overall results so far on benchmark functions and the proposed NACMM outperforms state-of-the-art flame segmentation approaches, providing a high detection accuracy and a low false detection rate.
\end{abstract}

\section{Introduction}

Nowadays, combustion furnaces have been widely applied in different fields of industry [1], such as coal-fired power plants [2], steelmaking [3], waste incineration [4], and cement production [5]. Since the combustion flame is one of the most direct characteristics that reflect the combustion status inside the industrial furnace, the accurate detection of combustion flame can effectively help operators adjust combustion strategies to improve combustion utilization and ensure safe operation. Therefore, various approaches were developed and applied to measure the combustion flame inside the industrial furnace [6-8], such as spectral analysis technology [6], optical fiber sensor technology [7], and temperature sensor technology [8].

With the development of machine vision, image processing technology has been gradually applied to detect and segment the combustion flame [9] because it has a high detection accuracy for flame segmentation. Wang et al. [10] present a new flame segmentation color model based on HSI and backpropagation, which transforms the RGB color image captured by the CCD into the HSI color image, and then the BP neural network is adopted to effectively segment the characteristics of combustion flame. Celik et al. [11] propose a generic flame segmentation color model that combines foreground object information with color pixel statistics of combustion flame, in which the foreground information is extracted by using adaptive background subtraction algorithm and then verified by the statistical flame color model to determine whether the segmented foreground object is a flame candidate information or not. Zhang et al. [12] use the nonlinear partial least-squares colorimetry and Monte Carlo with iterative optimization to research the actual combustion status for obtaining a flame distribution. Chen et al. [13] present a new color space model based on scale invariant feature transform (SIFT), in which the SIFT algorithm is introduced to extract the feature descriptors of combustion flame for achieving better 
adaptability and robustness model. Wang et al. [14] develop a convolutional neural network approach based on adaptive pooling, which effectively avoids the blindness in the traditional feature extraction process and the learning of invalid features in the convolutional neural network, and the experiment results show that the developed approach has a higher segmentation rate. Qiu et al. [15] propose an unsupervised classification measurement approach based on the convolutional autoencoder, in which the principal component analysis (PCA) and the hidden Markov model (HMM) are adopted to monitor the combustion condition with the uniformly spaced flame image. Zhang et al. [16] propose a new adaptive color model with a double threshold to improve the segmentation efficiency and detection accuracy of the combustion flame. Hashemzadeh and Zademehdi [17] develop a robust color model by using $\mathrm{K}$-medoids to reliably detect all candidate flame regions in a scene, which provides high detection accuracy and low false detection rate for flame recognition. Ye et al. [18] propose a new flame segmentation approach with wavelet analysis to detect smoke and flame simultaneously for color dynamic video sequences obtained. Bai et al. [19] use PCA and kernel support vector machine (KSVM) techniques to segment the combustion flame pixels, and the experimental results show that the PCA-KSVM model is feasible and effective in monitoring a combustion process. Han et al. [20] propose multicolor-based detection combining the RGB, HSI, and YUV color space which takes full advantage of the motion feature and color information of combustion flame.

As image segmentation contains multiple factors, it is difficult to obtain the best segmentation parameters by trial and error. Therefore, a variety of metaheuristic algorithms, such as particle swarm optimization [21], differential evolution [22], memetic algorithm [23], and genetic algorithm [24], have been adopted to search the optimal parameters for obtaining the best segmentation effect. However, the combustion states inside the industrial furnace, as well as the RGB components of combustion flame, change according to the production needs [25], which further challenges the optimal set of model parameters but is not considered in the previous works. To tackle this problem, the weight coefficients of RGB components and segmentation threshold are both considered as the variables to design the adaptive color model in this work. The segmentation threshold is the discrete number, which can be found out more efficiently by the binary-coding algorithm $[16,26]$; the weight coefficients are continuous variables between 0 and 1 , which are introduced to enhance the robustness of the model for combustion states. Thus, the objective function of the proposed adaptive color model is a hybrid-coding problem. The hybrid-coded human learning optimization (HcHLO) [27] is a novel and powerful framework for solving hybrid-coded problems, which achieved the so-far best-known results on a set of hybridcoded benchmark problems. Therefore, this paper proposes a novel segmentation method for furnace flame using adaptive color model and hybrid-coded HLO, in which a new adaptive color model with mixed variables (NACMM) is presented to effectively segment the flame pixels of different combustion states, and an adaptive hybrid-coded human learning optimization (AHcHLO) is developed to find the best optimized parameters of NACMM for guaranteeing the best performance. Regarding this proposed NACMM, two objective functions are adopted as the evaluation index to evaluate the segmentation accuracy and reduce the structural risk.

The rest of the paper is organized as follows. Section 2 presents the proposed AHcHLO in detail. Section 3 describes the furnace flame segmentation based on the NACMM with AHcHLO. Section 4 gives the performance comparison of the proposed AHcHLO with other recent algorithms and the comparison results of the segmentation simulation of furnace flame. Finally, conclusions are drawn in Section 5.

\section{Adaptive Hybrid-Coded Human Learning Optimization}

The HLO [28] algorithm adopts the three learning operators, i.e., the random learning operator (RLO), the individual learning operator (ILO), and the social learning operator (SLO), to search for the optimal solution. Nowadays, HLO has been successfully used to solve the various types of optimization problems, such as furnace flame recognition [16], image segmentation [26], knapsack problems [29], engineering design problems [27, 30], optimal power flow calculation [31], extractive text summarization [32], financial markets forecasting [33], scheduling problems [34], and intelligent control [35]. To solve the mixed variables of NACMM more effectively, an adaptive strategy is developed to further enhance the search ability of AHcHLO.

2.1. Initialization. Like the standard HcHLO [27], the proposed AHcHLO adopts the binary-real mixed coding framework to represent the individual's knowledge, in which continuous parameters are directly represented as realcoded variables, which are randomly initialized between the lower bound and upper bound, while the Boolean or discrete parameters are coded as a binary string, which is stochastically initialized with " 0 " or " 1 ." Thus, an individual of $\mathrm{AHcHLO}$ is represented as

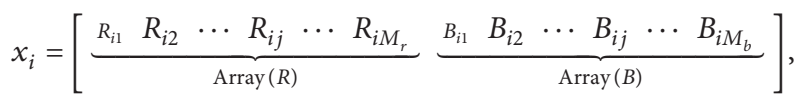

$$
\begin{aligned}
& 1 \leq i \leq N \text {, }
\end{aligned}
$$

where $x_{i}$ denotes the $i$-th individual; $\operatorname{Array}(R)$ and $\operatorname{Array}(B)$ store the real-coded variables and the binary/discrete variables of solutions, respectively. $N$ is the size of population, and $M_{r}$ and $M_{b}$ denote the lengths of the real-coded variables and binary strings, respectively. The whole dimension of solutions is $M$, and $M=M_{r}+M_{b}$. Initially, the elements of each individual in $\operatorname{Array}(R)$ and $\operatorname{Array}(B)$ are randomly initialized. After generating $N$ individuals, an initial population is obtained as 


$$
X=\left[\begin{array}{c}
x_{1} \\
x_{2} \\
\vdots \\
x_{i} \\
\vdots \\
x_{N}
\end{array}\right]=\left[\begin{array}{cccccccccccc}
R_{11} & R_{12} & \cdots & R_{1 j} & \cdots & R_{1 M_{r}} & B_{11} & B_{12} & \cdots & B_{1 j} & \cdots & B_{1 M_{b}} \\
R_{21} & R_{22} & \cdots & R_{2 j} & \cdots & R_{2 M_{r}} & B_{21} & B_{22} & \cdots & R_{2 j} & \cdots & B_{2 M_{b}} \\
\vdots & \vdots & & \vdots & & \vdots & \vdots & \vdots & & \vdots & & \vdots \\
R_{i 1} & R_{i 2} & \cdots & R_{i j} & \cdots & R_{i M_{r}} & B_{i 1} & B_{i 2} & \cdots & B_{i j} & \cdots & B_{i M_{b}} \\
\vdots & \vdots & & \vdots & & \vdots & \vdots & \vdots & & \vdots & \vdots \\
R_{N 1} & R_{N 2} & \cdots & R_{N j} & \cdots & R_{N M_{r}} & \underbrace{B_{N 1}}_{\text {Array }(R)} & B_{N 2} & \cdots & B_{N j} & \cdots & B_{N M_{b}}
\end{array}\right] .
$$

\subsection{Learning Operators}

2.2.1. Random Learning Operator. Random learning [36] usually exists in human learning as there is no prior knowledge of problems at the beginning. With the progress of learning, the random learning strategy remains to keep the peculiar creativity of human beings. Inspired by the random learning strategy, the random learning operator (RLO) is used in AHcHLO, in which real-coding variables $R_{i j}$ are operated by (3) while the bits of binary strings are generated by (4).

$$
\begin{aligned}
& R_{i j}=x_{\min , j}+r_{1} \times\left(x_{\max , j}-x_{\min , j}\right), \\
& B_{i j}=\operatorname{Rand}(0,1)= \begin{cases}0, & 0 \leq r_{2} \leq 0.5, \\
1, & \text { else, }\end{cases}
\end{aligned}
$$

where $x_{\min , j}$ and $x_{\max , j}$ are the lower bound and upper bound of real-coded variable $j ; r_{1}$ and $r_{2}$ are two independent random numbers between 0 and 1 .

2.2.2. Individual Learning Operator. Individual learning [37] is an efficient learning strategy by adopting the obtained personal experience to avoid the same mistakes. To imitate the individual learning strategy, the personal best solutions are saved in the individual knowledge database (IKD) of $\mathrm{AHcHLO}$, which is represented as

$$
I K D=\left[\begin{array}{c}
i k d_{1} \\
i k d_{2} \\
\vdots \\
i k d_{i} \\
\vdots \\
i k d_{N}
\end{array}\right], \quad 1 \leq i \leq N
$$

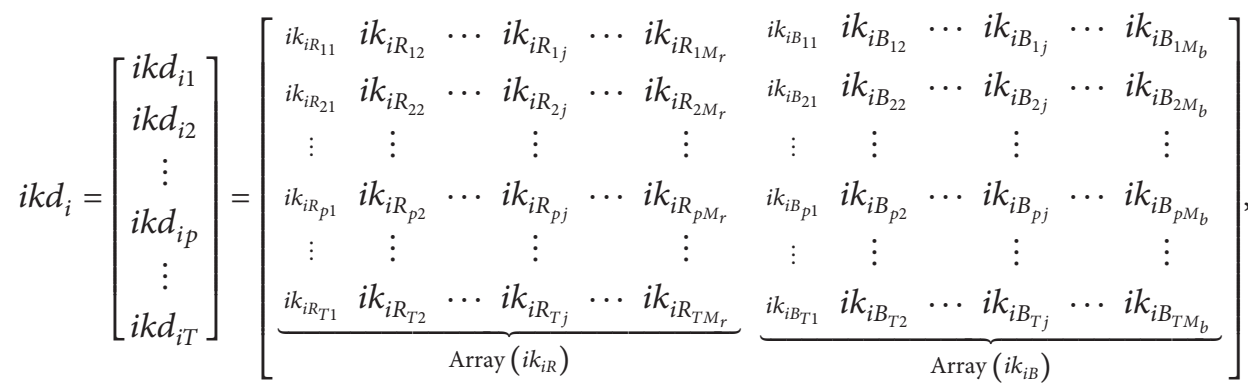

where $i k d_{i}$ stands for the IKD of individual $i, i k_{i R_{p j}} / i k_{i B_{p j}}$ are the $j$-th real-coded/binary knowledge of $p$-th best solution of individual $i$, and $T$ is the size of $i k d_{i}$.

When AHcHLO runs the individual learning operator (ILO), the linear individual learning operator (IILO) is used to handle the real-coding knowledge in $\operatorname{Array}\left(i k_{i R}\right)$ as (7), and the standard individual learning operator for HLO is adopted to deal with the bits in $\operatorname{Array}\left(i k_{i B}\right)$ as (8).

$$
\begin{aligned}
& R_{i j}=i k_{i R_{p j}}+I_{L} \times r_{3} \times\left(s k_{q R_{j}}-i k_{i R_{p j}}\right), \\
& B_{i j}=i k_{i B_{p j}},
\end{aligned}
$$

where $I_{L}$ is the linear individual learning factor and $r_{3}$ is a stochastic number between -1 and 1 . 
2.2.3. Social Learning Operator. Social learning [38] plays an important role in an integrated social environment because it allows humans to copy the best information in the population, and therefore it can greatly improve learning efficiency and effectiveness. To imitate the social learning strategy, the best knowledge of population is stored in the social knowledge data $(S K D)$ as

$$
S K D=\left[\begin{array}{c}
s k d_{1} \\
s k d_{2} \\
\vdots \\
s k d_{q} \\
\vdots \\
s k d_{H}
\end{array}\right]=\left[\begin{array}{cccccccccccc}
s k_{1 R_{1}} & s k_{1 R_{2}} & \cdots & s k_{1 R_{j}} & \cdots & s k_{1 R_{M_{r}}} & s k_{1 B_{1}} & s k_{1 B_{2}} & \cdots & s k_{1 B_{j}} & \cdots & s k_{1 B_{M_{b}}} \\
s k_{2 R_{1}} & s k_{2 R_{2}} & \cdots & s k_{2 R_{j}} & \cdots & i k_{2 R_{M_{r}}} & s k_{2 B_{1}} & s k_{2 B_{2}} & \cdots & s k_{2 B_{j}} & \cdots & s k_{2 B_{M_{b}}} \\
\vdots & \vdots & & \vdots & & \vdots & \vdots & \vdots & & \vdots & & \vdots \\
s k_{q R_{1}} & s k_{q R_{2}} & \cdots & s k_{q R_{j}} & \cdots & s k_{q R_{M_{r}}} & s k_{q B_{1}} & s k_{q B_{2}} & \cdots & s k_{q B_{j}} & \cdots & s k_{q B_{M_{b}}} \\
\vdots & \vdots & & \vdots & & \vdots & \vdots & \vdots & & \vdots & \vdots \\
s k_{H R_{1}} & s k_{H R_{2}} & \cdots & s k_{H R_{j}} & \cdots & s k_{H R_{M_{r}}} & \underbrace{s k_{H B_{1}}}_{\text {Array }\left(s k_{R}\right)} & s k_{H B_{2}} & \cdots & s k_{H B_{j}} & \cdots & s k_{H B_{M_{b}}}
\end{array}\right],
$$

where $s k_{q R_{j}} / s k_{q B_{j}}$ denote the $j$-th real-coded/binary knowledge of $q$-th best solution in the SKD, and $H$ is the size of SKD.

When AHcHLO performs the social learning operator (SLO) to generate new candidate solutions, the linear social learning operator (ISLO) is used to operate the continuous variables in $\operatorname{Array}\left(s k_{R}\right)$ as (10), and the standard social learning operator in HLO is adopted to deal with the bits in $\operatorname{Array}\left(s k_{B}\right)$ as $(11)$.

$$
\begin{aligned}
& R_{i j}=s k_{q R_{j}}+S_{L} \times r_{4} \times\left(s k_{q R_{j}}-i k_{i R_{p j}}\right), \\
& B_{i j}=s k_{q B_{j}},
\end{aligned}
$$

where $S_{L}$ stands for the linear social learning factor and $r_{4}$ is a random number between 0 and 1 .

2.3. Adaptive Strategy. Obviously, the linear individual learning factor $I_{L}$ and the linear social learning factor $S_{L}$ are extremely important because they directly determine the learning abilities of IILO and ISLO, respectively. In the standard HcHLO, these two parameters, i.e., $I_{L}$ and $S_{L}$, are both set as constants, and the recommended values are 1 and 2; that is, the individual always learns and solves problems with the same capability or level of proficiency, which is not true for real human learning. Qamar et al. [39] points out that the learning strategies of humans change as the quality of the sensory evidence varies, and Zimmerman and Martinez-Pons [40] indicate that both convergent and discriminative validity exist in the construct of human learning. Humans usually search for the best possible knowledge in a wide range as they lack prior knowledge of problems at the beginning [41], and they adjust and reduce their learning strategies to approach optimal knowledge based on the last learning result with the progress of learning [40]. As Scarbrough et al. [42] state, the adaptive learning strategy can effectively exploit the accumulated knowledge of human beings in situations that are uncertain and complicated. Inspired by these discoveries, the adaptive strategies for $I_{L}$ and $S_{L}$ are developed in AHcHLO to improve the search efficiency and solution quality of the algorithm, which is presented as

$$
\begin{gathered}
I_{L}=I_{L \max }-\frac{I_{L \text { max }}-I_{L \text { min }}}{\text { Ite }_{\text {max }}} \times \text { Ite }, \\
S_{L}=S_{L \text { max }}-\frac{S_{L \text { max }}-S_{L \text { min }}}{\text { Ite }_{\text {max }}} \times \text { Ite },
\end{gathered}
$$

where $I_{L \min } / S_{L \min }$ and $I_{L \max } / S_{L \max }$ are the minimum and maximum values of $I_{L} / S_{L}$, respectively; Ite and Ite $e_{\max }$ are the current iteration number and maximum iteration number of searches, respectively.

With the introduction of the adaptive $I_{L}$ and $S_{L}$ strategy, the proposed AHcHLO can efficiently explore the interesting solution areas more widely at the beginning of iterations, search for the optimal candidate solution in a more suitable range at the middle of the search process, and perform the accurate local search to find the optima at the end of generations. Therefore, the proposed AHcHLO can achieve a practically ideal trade-off between exploration and exploitation, and the optimization search ability of AHcHLO is significantly enhanced.

2.4. Updating of the IKD and the SKD. Since AHcHLO is designed for solving single-objective problems, the sizes of IKDs and SKD are both set to 1 as recommended in [43]. After a new population is generated, the fitness values of all individuals are calculated according to the predefined fitness function to update the IKDs. The new candidate replaces the original solution in the IKDs only if its fitness value is superior. Otherwise, the original solution in the current IKDs will remain. Similarly, the new candidate is saved to replace the current one in the SKD only if it has a better fitness value. Besides, the $I K D$ is reinitialized to further enhance the diversity if it is not updated in 100 generations as [27].

2.5. Implementation of $\mathrm{AHcHLO}$. In summary, AHcHLO uses three learning operators, i.e., the random learning 
operator (RLO), the adaptive individual learning operator (AILO), and the adaptive social learning operator (ASLO), to yield new candidates to search for the optimal solution, which can be summarized as

$$
x_{i j}= \begin{cases}\text { RLO, } & 0 \leq r<p r, \\ \text { AILO, } & p r \leq r<p i, \\ \text { ASLO, } & p i \leq r<1,\end{cases}
$$

where $r$ is a random number from 0 to $1 ; p r,(p i-p r)$, and $(1-p i)$ are the probabilities of running RLO, AILO, and ASLO, respectively.

The implementation of AHcHLO can be concluded as follows, and the pseudocode for AHcHLO is shown in Algorithm 1.

Step 1: initialize the population randomly; set the control parameters of AHcHLO, which mainly includes the size of population, Ite $e_{\max }, p r, p i, I_{L \min }, I_{L \max }, S_{L \text { min }}$ and $S_{L \max }$

Step 2: calculate the fitness values of all initial individuals and initialize the IKDs and SKD

Step 3: perform RLO, AILO, and ASLO as (14) to generate new candidate solutions

Step 4: calculate the fitness values of all new candidate individuals

Step 5: update the IKDs and SKD according to the updating rules

Step 6: calculate the linear individual learning factor $I_{L}$ and the linear social learning factor $S_{L}$ according to the developed adaptive strategies as (12) and (13)

Step 7: if the termination conditions are met, output the best solution; otherwise go to Step 3

\section{Furnace Flame Segmentation Based on the NACMM with AHcHLO}

3.1. Basic Idea of NACMM. The color information is the most obvious characteristic of combustion flame, which can effectively reflect the flame temperature of different combustion states. The previous works $[44,45]$ point out that the flame temperature is related to the RGB gray values. When the temperature is relatively low, the $\mathrm{R}$ gray values are large while the $G$ and $B$ gray values are both small, and the flame color appears orange. With the temperature increasing, the $G$ gray values gradually increase and the flame color becomes brighter. When the flame temperature further increases, the B gray values also increase and the flame color becomes pure white. The four flame images of different combustion states are shown in Figure 1; the flame temperatures in Figures 1(c) and 1(d) are greater than that in Figure 1(b), and the flame temperature in Figure 1(b) is greater than that in Figure 1(a). In the traditional flame segmentation algorithm, the average method is often used to preprocess the original image, which is shown as

$$
g(x, y)=k_{r} \times f_{R}(x, y)+k_{g} \times f_{G}(x, y)+k_{b} \times f_{B}(x, y),
$$

where $g(x, y)$ represents the gray value of grayed image in the pixel position $(x, y) ; f_{R}(x, y), f_{G}(x, y)$, and $f_{B}(x, y)$ are the RGB gray values of the original image in the pixel position $(x, y)$, respectively; and $k_{r}, k_{g}$, and $k_{b}$ are the weight coefficients of RGB components, and $k_{r}=k_{g}=k_{b}=(1 / 3)$.

The relationship between combustion states and RGB gray values is not considered in the previous works, which influences the segmentation accuracy of combustion flame. To better achieve the separation of flame pixels, the adaptive weight coefficients are considered in this work. Moreover, the five image graying methods, i.e., average method $\left(k_{r}=k_{g}=k_{b}=(1 / 3)\right)$, maximum method (selecting the maximum gray values of RGB components), weighted average method $1\left(k_{r}=0.7, k_{g}=0.15, k_{b}=0.15\right)$, weighted average method $2 \quad\left(k_{r}=0.15, k_{g}=0.7, k_{b}=0.15\right)$, and weighted average method $3\left(k_{r}=0.15, k_{g}=0.15, k_{b}=0.7\right)$, are used to gray flame images I-IV for intuitively understanding these characteristics, which are shown in Figures 2-5. By comparing the characteristics of flame images I-IV under different image graying methods, the following conclusions can be made:

(1) Figure 2 clearly confirms that the maximum method obtains an ideal grayscale image because the $f_{R}(x, y)$ gray values are the largest, the $f_{G}(x, y)$ gray values are the second, and the $f_{B}(x, y)$ gray values are the least. The maximum method is also the special case of the weighted average method; i.e., $k_{r}=1, k_{g}=0, k_{b}=0$.

(2) Figure 3 demonstrates that weighted average method 2 achieves the best grayscale image because the color of combustion flame becomes brighter. Meanwhile, compared with weighted average method 1, weighted average method 2 reduces the interference of external light.

(3) Figure 4 explicitly proves that weighted average method 3 obtains the best grayscale image, which effectively reduces the interference of R gray values around the combustion flame. In particular, for the maximum method, Figure 4(b) does not benefit from the separation of flame pixels.

(4) Figure 5 effectively confirms that the effects of grayscale image under the five methods are almost the same because the color of combustion flame is white; that is, the RGB gray values are all close to the maximum, and there is no color interference of external light.

3.2. New Adaptive Color Model with Mixed Variables. The above comparison results clearly show that the flame images of different combustion states prefer different image graying methods to obtain the best effect of grayscale images because of the change of RGB gray values of combustion flame. Specifically, the $\mathrm{R}$ gray values are very significant for discriminating between "flame" and "nonflame" regions when the temperature is relatively low, and the G and B gray values are more conducive to segmenting the flame pixels with the increase of the temperature. Therefore, a new adaptive color 


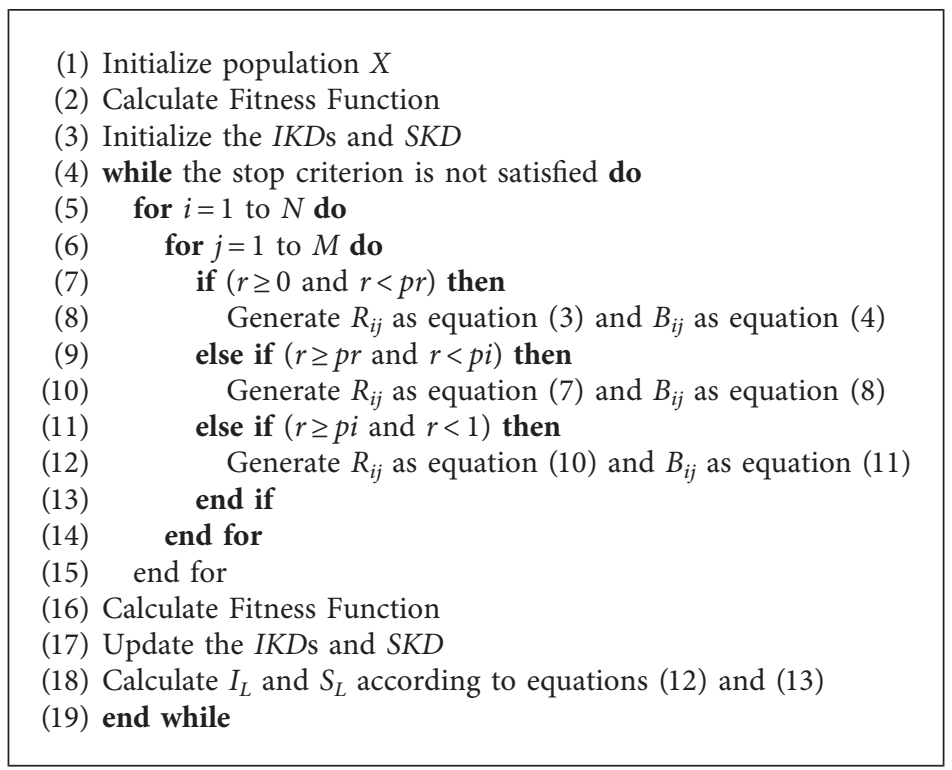

Algorithm 1: Pseudocode for AHcHLO.

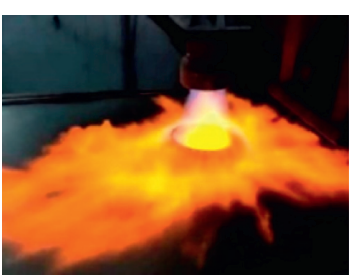

(a)

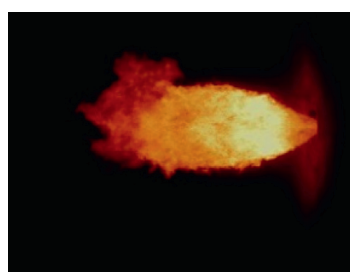

(b)

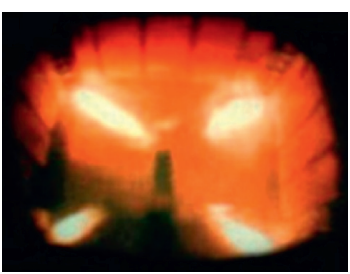

(c)

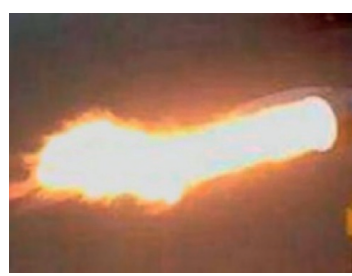

(d)

FIgURE 1: The four flame images of different combustion states: (a) I; (b) II; (c) III; (d) IV.

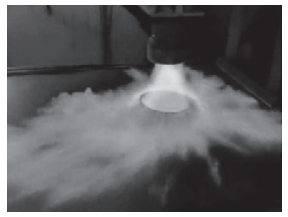

(a)

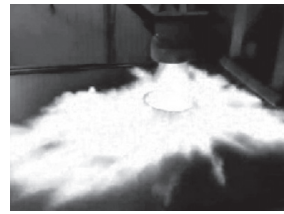

(b)

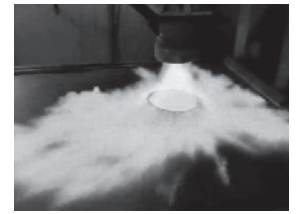

(c)

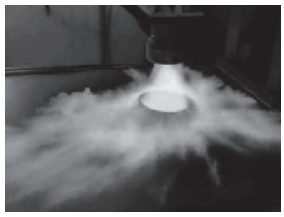

(d)

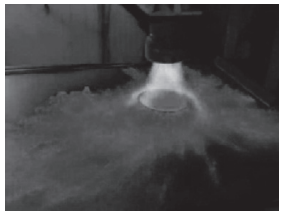

(e)

Figure 2: The comparison results of flame image I under different methods: (a) average method; (b) maximum method; (c) weighted average method 1; (d) weighted average method 2; (e) weighted average method 3.

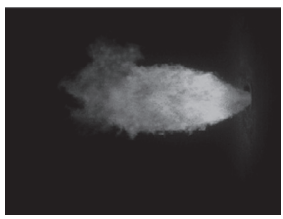

(a)

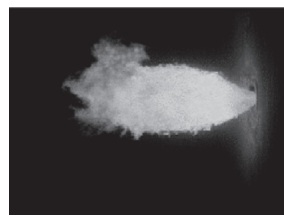

(b)

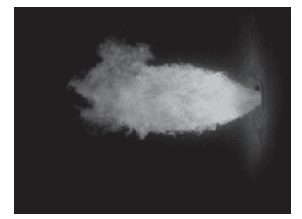

(c)

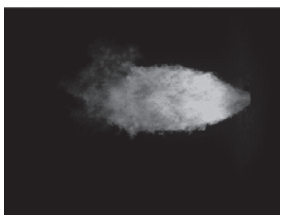

(d)

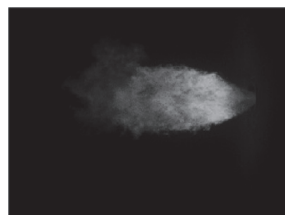

(e)

FIgURE 3: The comparison results of flame image II under different methods: (a) average method; (b) maximum method; (c) weighted average method 1 ; (d) weighted average method 2 ; (e) weighted average method 3. 


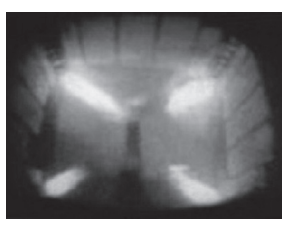

(a)

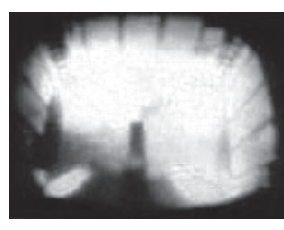

(b)

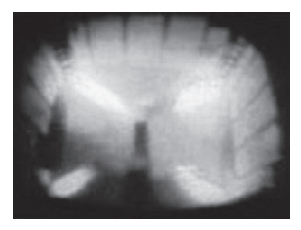

(c)

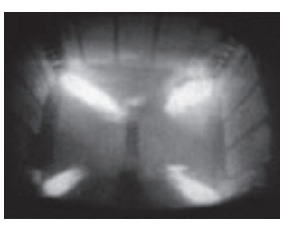

(d)

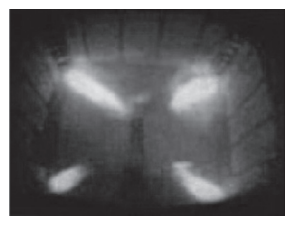

(e)

Figure 4: The comparison results of flame image III under different methods: (a) average method; (b) maximum method; (c) weighted average method 1 ; (d) weighted average method 2; (e) weighted average method 3.

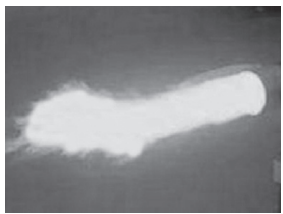

(a)

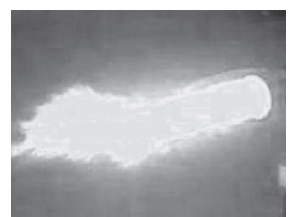

(b)

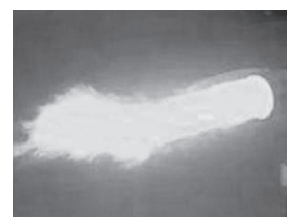

(c)

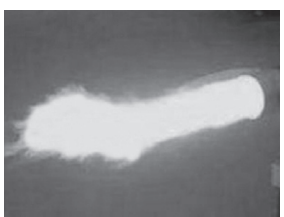

(d)

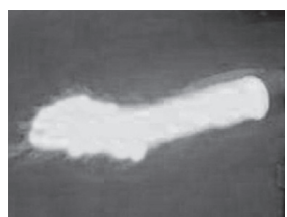

(e)

FIGURE 5: The comparison results of flame image IV under different methods: (a) average method; (b) maximum method; (c) weighted average method 1; (d) weighted average method 2; (e) weighted average method 3.

model with mixed variables (NACMM) is proposed for adapting to the different combustion states, in which the weight coefficients of RGB components, i.e., $k_{R}, k_{G}$, and $k_{B}$, and segmentation threshold $t_{1}$ are simultaneously adjusted.

For a given flame image in NACMM, the corresponding grayed image $g_{1}$ is obtained by the adaptive weighted average method as (16), in which the median filtering method, i.e., (17), is adopted to effectively smooth the interference points and maintain the edge information of combustion flame because of the noise interference factors in the original image.

$$
\begin{gathered}
g_{1}(x, y)=k_{R} \times f_{2 R}(x, y)+k_{G} \times f_{2 G}(x, y)+k_{B} \times f_{2 B}(x, y), \\
\left\{\begin{array}{l}
f_{2 R}(x, y)=\operatorname{median}\left\{f_{R}(x+s, y+s)\right\} \\
f_{2 G}(x, y)=\operatorname{median}\left\{f_{G}(x+s, y+s)\right\}, \quad-\frac{n_{1}-1}{2} \leq s \leq \frac{n_{1}-1}{2}, \\
f_{2 B}(x, y)=\operatorname{median}\left\{f_{B}(x+s, y+s)\right\}
\end{array}\right.
\end{gathered}
$$

where $k_{R}, k_{G}$, and $k_{B}$ are the weight coefficients of RGB components, and $k_{R}+k_{G}+k_{B}=1 ; f_{2 R}(x, y), f_{2 G}(x, y)$, and $f_{2 B}(x, y)$ are the RGB gray values of the filtered flame image in the pixel position $(x, y)$, respectively; and $n_{1}$ is the size of filter window and is set to 5 in this work.

Then, the preset threshold variable $t_{1}$ is used to effectively segment the grayed image $g_{1}$, and the flame/nonflame pixels are marked as white/black pixels, respectively, which is presented as

$$
g_{2}(x, y)= \begin{cases}1, & \text { if } g_{1}(x, y)>t_{1} \\ 0, & \text { else }\end{cases}
$$

where $g_{2}$ represents the processing result of the segmented flame image.

In NACMM, the preset weight coefficients $k_{R}, k_{G}$, and $k_{B}$ are continuous distribution optimization problems between 0 and 1 , and the preset threshold variable $t_{1}$ is a discrete variable optimization problem between 0 and 255. The previous works $[16,26]$ prove that the binary-coding algorithm can calculate the segmentation threshold $t_{1}$ more efficiently; that is, the objective function of NACMM is a hybrid-coding problem.

3.3. The NACMM with AHcHLO. Then, the proposed AHcHLO is performed to search for the best values of the parameters $t_{1}, k_{R}, k_{G}$, and $k_{B}$. The objective function $\mathrm{Obj}_{1}$ is calculated by the sample pixels to evaluate the quality of NACMM. Note that the NACMM optimized only by using $\mathrm{Obj}_{1}$ still has a structural risk because of the limitation of the number of sample pixels. Specifically, the preset threshold $t_{1}$ within a certain range $\left[T_{1}, T_{2}\right]$ can achieve the separation of sample pixels, but the limit thresholds $T_{1}$ and $T_{2}$ cannot effectively separate the pixels on the edge of combustion flame. To effectively reduce this structural risk, the objective function $\mathrm{Obj}_{2}$ is adopted to further optimize the preset threshold $t_{1}$ for obtaining better NACMM when the values of objective function $\mathrm{Obj}_{1}$ are equal. Therefore, the best NACMM with optimal parameters $t_{1}, k_{R}, k_{G}$, and $k_{B}$ is obtained through the evaluation of objective functions $\mathrm{Obj}_{1}$ and $\mathrm{Obj}_{2}$. 
3.3.1. Construction of Objective Function $\mathrm{Obj}_{1}$. The objective function $\mathrm{Obj}_{1}$ is the proportion of the number of correctly classified sample pixels (flame and nonflame) to the total number of sample pixels, and the higher value of $\mathrm{Obj}_{1}$ indicates better NACMM, which is given by

$$
\operatorname{Obj}_{1}\left(t_{1}, k_{R}, k_{G}, k_{B}\right)=\frac{N_{1}+N_{2}}{M_{1}+M_{2}} \times 100 \%,
$$

where $N_{1}$ and $N_{2}$ are the number of correctly classified flame and nonflame sample pixels, respectively; $M_{1}$ and $M_{2}$ represent the total number of flame and nonflame sample pixels, respectively. The flame/nonflame pixels from the filtered flame image are extracted to construct the flame/nonflame sample pixels, respectively. As the sample pixels have a greater impact on the quality of NACMM, they are manually selected from different parts of the flame/nonflame regions. The examples of selected flame/nonflame sample pixels for flame image I are given in Figures 6(b) and 6(c), in which the size of each selected sample image is $n_{2} \times n_{2}$, the flame/ nonflame sample images are selected 20 times, respectively, and therefore $M_{1}=M_{2}=20 \times n_{2} \times n_{2}$.

3.3.2. Construction of Objective Function $\mathrm{Obj}_{2}$. For the grayed image $g_{1}$, the total intensity levels can be expressed as $L$ lying in the range $\left[t_{0}, t_{2}\right]$ where $t_{0}$ and $t_{2}$ are the minimum and maximum gray intensity levels, respectively. Further, let the pixel number of $L$ intensity level and all intensity levels be denoted as $E_{L}$ and $E$, respectively. Because there are only two classes of pixels for the flame image, i.e., the nonflame pixels and the flame pixels, the flame pixels in $g_{1}$ can be efficiently segmented and extracted based on their gray intensity levels and the preset threshold variable $t_{1}$; that is, the nonflame pixels are from $t_{0}$ to $t_{1}$, and the flame pixels are from $t_{1}$ to $t_{2}$. Therefore, the objective function $\mathrm{Obj}_{2}$ is calculated by the Otsu method as (20)-(23), and the higher value of $\mathrm{Obj}_{2}$ indicates better NACMM.

$$
\begin{aligned}
\mathrm{Obj}_{2}\left(t_{1}\right) & =\sum_{c=1}^{2} p_{c}\left(t_{1}\right) \times\left[u_{c}\left(t_{1}\right)-u_{C}\right]^{2}, \\
u_{c}\left(t_{1}\right) & =\sum_{L=t_{c-1}}^{t_{c}} \frac{L \times\left(E_{L} / E\right)}{p_{c}\left(t_{1}\right)} \\
p_{c}\left(t_{1}\right) & =\sum_{L=t_{c-1}}^{t_{c}} \frac{E_{L}}{E} \\
u_{C} & =\sum_{L=t_{0}}^{t_{2}} L \times \frac{E_{L}}{E}
\end{aligned}
$$

where $c$ denotes the class of pixels, $c \in\{1,2\}$; that is, $c=1$ and $c=2$ are the classes of nonflame pixels and flame pixels, respectively; $u_{c}\left(t_{1}\right)$ and $p_{c}\left(t_{1}\right)$ represent the probability of mean and occurrence of class $c$, respectively; and $u_{C}$ is the total mean of all classes.
In summary, the NACMM with AHcHLO is optimized by two objective functions for improving the segmentation accuracy and reducing the structural risk. Firstly, the flame/ nonflame sample pixels are constructed from the flame image, and the segmentation threshold $t_{1}$ and the weight coefficients, i.e., $k_{R}, k_{G}, k_{B}$, are initialized as binary strings and real-coded variables, respectively. On this basis, the filtered flame image is segmented based on the initialized parameters, and the corresponding objective function values $\mathrm{Obj}_{1} / \mathrm{Obj}_{2}$ are calculated. Then, the AHcHLO is used to generate new candidate parameters, and the corresponding candidate objective function value $\mathrm{Obj}_{1}^{\prime}$ is calculated to evaluate the quality of NACMM. The new candidate is saved to replace the current one in the IKDs and SKD only if it has a better value of the objective function. Note that the corresponding objective function $\mathrm{Obj}_{2}^{\prime}$ needs to be calculated and compared with $\mathrm{Obj}_{2}$ if the value of objective function $\mathrm{Obj}_{1}$ is equal to that of $\mathrm{Obj}_{1}^{\prime}$, which can further obtain better threshold $t_{1}$ for reducing the structural risk of NACMM. When the termination conditions are met, the optimal parameters $t_{1}, k_{R}, k_{G}$, and $k_{B}$ are output to obtain the best NACMM. Finally, the best NACMM is adopted to segment the flame image, and the flame/nonflame pixels are marked as white/black pixels, respectively. The furnace flame segmentation based on the NACMM with AHcHLO is summarized in Figure 7.

\section{Experimental Results and Discussion}

In this section, the proposed AHcHLO was firstly used to solve the benchmark functions for evaluating its optimization ability. Then, the segmentation simulation for furnace flame based on the NACMM with AHcHLO was performed to verify its effectiveness and feasibility.

4.1. The Benchmark Functions. A total of 14 optimization problems with mixed variables [27] were adopted as the benchmark functions to evaluate the performance of the proposed AHcHLO, and the numerical results of AHcHLO were compared with the five recent optimization algorithms, i.e., hybrid-coded human learning optimization algorithm (HcHLO) [27], adaptive simplified human learning optimization algorithm (ASHLO) [43], improved adaptive human learning optimization algorithm (IAHLO) [30], scalefree particle swarm optimization (SFPSO) [46], and hybrid particle swarm optimization with adaptive learning (ALPSO) [47]. A set of fair parameters obtained by a simple trial-and-error procedure was adopted for $\mathrm{AHcHLO}$, that is, $p r=0.1, p i=0.85, I_{L \max }=2.0, I_{L \min }=0.2, S_{L \max }=3.0$, and $S_{L \min }=1.0$. The population size and the maximal iteration number of AHcHLO were set as those recommended in [27]. For a fair comparison, HcHLO, ASHLO, IAHLO, SFPSO, and ALPSO used the recommended parameter values, and the maximal number of function calculations was the same as that of AHcHLO. Besides, if the gap between the found one and the theoretical optima is less than $10^{-6}$, the search will be terminated as suggested in [48]. All the cases ran 100 times independently. 


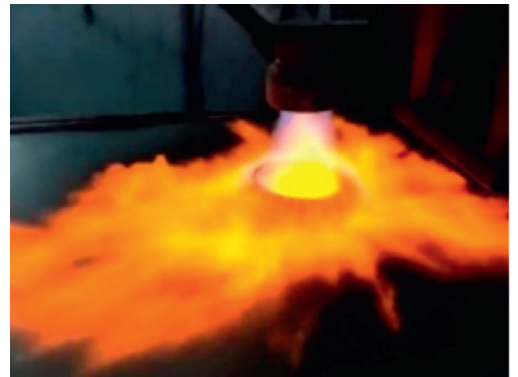

(a)

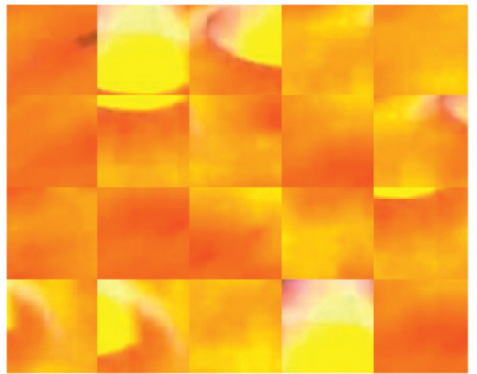

(b)

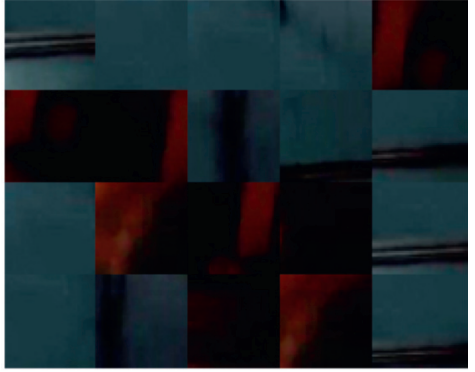

(c)

Figure 6: Extracting sample pixels from flame image I: (a) original flame image; (b) flame sample pixels; (c) nonflame sample pixels.

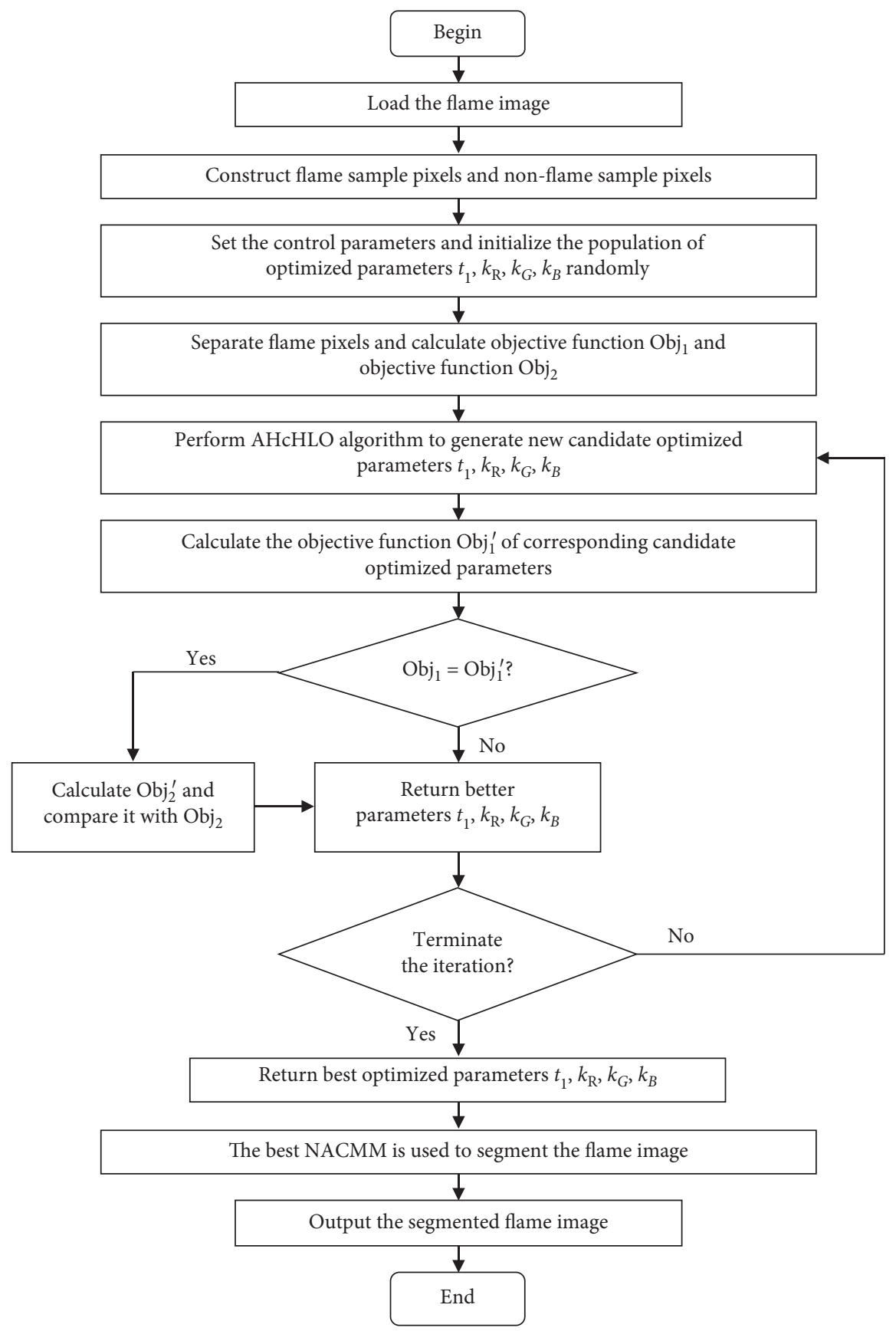

FIGURE 7: Flowchart of proposed furnace flame segmentation method. 
TABle 1: Results of AHcHLO, HcHLO, ASHLO, IAHLO, SFPSO, and ALPSO on the benchmark functions.

\begin{tabular}{|c|c|c|c|c|c|c|c|}
\hline Fun & Metric & AHcHLO & HcHLO & ASHLO & IAHLO & SFPSO & ALPSO \\
\hline \multirow{5}{*}{$\mathrm{F} 1$} & Best & 87.500000 & 87.500000 & 87.500000 & 87.500000 & 87.500000 & 87.500000 \\
\hline & Mean & 87.500000 & 87.500000 & 87.500000 & 87.500000 & 87.500000 & 87.500000 \\
\hline & Std & $0.00 E+00$ & $3.02 E-08$ & $0.00 E+00$ & $0.00 E+00$ & $0.00 E+00$ & $0.00 E+00$ \\
\hline & $t$-test & - & - & 0 & 0 & 0 & 0 \\
\hline & $W$-test & - & - & 0 & 0 & 0 & 0 \\
\hline \multirow{5}{*}{ F2 } & Best & 7.667180 & 7.667181 & 7.667180 & 7.667180 & 7.667180 & 7.667180 \\
\hline & Mean & 7.667180 & 7.667194 & 7.667180 & 7.667180 & 7.667180 & 7.667180 \\
\hline & Std & $1.78 E-15$ & $4.76 E-06$ & $1.78 E-15$ & $1.78 E-15$ & $1.78 E-15$ & $1.78 E-15$ \\
\hline & $t$-test & - & - & 0 & 0 & 0 & 0 \\
\hline & $W$-test & - & - & 0 & 0 & 0 & 0 \\
\hline \multirow{5}{*}{ F3 } & Best & 4.579582 & 4.579587 & 4.579636 & 4.580169 & 4.579592 & 4.670711 \\
\hline & Mean & 4.579584 & 4.579597 & 4.611342 & 4.611562 & 4.589737 & 5.017949 \\
\hline & Std & $1.65 E-06$ & $3.02 E-06$ & $7.17 E-02$ & $5.14 E-02$ & $4.27 E-02$ & $2.26 E-01$ \\
\hline & $t$-test & - & - & 1 & 1 & 1 & 1 \\
\hline & $W$-test & - & - & 1 & 1 & 1 & 1 \\
\hline \multirow{5}{*}{ F4 } & Best & 2.000000 & 2.000000 & 2.000001 & 2.000001 & 2.000001 & 2.000000 \\
\hline & Mean & 2.000000 & 2.000000 & 2.000001 & 2.001420 & 2.000001 & 2.002490 \\
\hline & Std & $0.00 E+00$ & $2.81 E-07$ & $4.44 E-16$ & $1.40 E-02$ & $4.44 E-16$ & $2.35 E-02$ \\
\hline & $t$-test & - & - & 1 & 0 & 1 & 0 \\
\hline & $W$-test & - & - & 1 & 1 & 1 & 0 \\
\hline \multirow{5}{*}{ F5 } & Best & 2.124468 & 2.124470 & 2.124469 & 2.124469 & 2.124469 & 2.124468 \\
\hline & Mean & 2.124488 & 2.124470 & 2.133572 & 2.142858 & 2.124567 & 2.133234 \\
\hline & Std & $2.03 E-04$ & $6.42 E-07$ & $6.07 E-02$ & $8.48 E-02$ & $2.42 E-04$ & $6.07 E-02$ \\
\hline & $t$-test & - & - & 0 & 1 & 1 & 0 \\
\hline & $W$-test & - & - & 1 & 1 & 1 & 0 \\
\hline \multirow{5}{*}{ F6 } & Best & 1.076543 & 1.076546 & 1.102035 & 1.108898 & 1.103897 & 1.077931 \\
\hline & Mean & 1.076544 & 1.081757 & 1.241141 & 1.239521 & 1.235834 & 1.129488 \\
\hline & Std & $5.25 E-06$ & $2.97 E-02$ & $6.82 E-02$ & $6.54 E-02$ & $6.22 E-02$ & $3.02 E-02$ \\
\hline & $t$-test & - & - & 1 & 1 & 1 & 1 \\
\hline & $W$-test & - & - & 1 & 1 & 1 & 1 \\
\hline \multirow{5}{*}{ F7 } & Best & 99.239640 & 99.239635 & 99.239640 & 99.239640 & 99.239640 & 99.239640 \\
\hline & Mean & 99.239640 & 99.241553 & 102.469891 & 101.291827 & 99.325068 & 99.240113 \\
\hline & Std & $1.42 E-14$ & $1.71 E-03$ & $3.91 E+00$ & $3.32 E+00$ & $8.50 E-01$ & $3.47 E-03$ \\
\hline & $t$-test & - & - & 1 & 1 & 0 & 0 \\
\hline & $W$-test & - & - & 1 & 1 & 0 & 1 \\
\hline \multirow{5}{*}{ F8 } & Best & 3.557461 & 3.557466 & 3.558204 & 3.557911 & 3.607595 & 3.570644 \\
\hline & Mean & 3.559827 & 3.558935 & 3.585373 & 3.592501 & 3.607832 & 3.665221 \\
\hline & Std & $6.56 E-03$ & $4.88 E-03$ & $3.88 E-02$ & $4.15 E-02$ & $6.88 E-04$ & $6.32 E-02$ \\
\hline & $t$-test & - & - & 1 & 1 & 1 & 1 \\
\hline & $W$-test & - & - & 1 & 1 & 1 & 1 \\
\hline \multirow{5}{*}{ F9 } & Best & -32217.430000 & -32217.42778 & -32217.430000 & -32217.430000 & -32217.430000 & -32217.430000 \\
\hline & Mean & -32217.430000 & -32217.42778 & -32217.430000 & -32217.430000 & -32217.430000 & -32217.430000 \\
\hline & Std & $3.64 E-12$ & $2.19 E-11$ & $3.64 E-12$ & $3.64 E-12$ & $3.64 E-12$ & $3.64 E-12$ \\
\hline & $t$-test & - & - & 0 & 0 & 0 & 0 \\
\hline & $W$-test & - & - & 0 & 0 & 0 & 0 \\
\hline \multirow{5}{*}{ F10 } & Best & -0.808844 & -0.808844 & -0.808844 & -0.808844 & -0.808844 & -0.726114 \\
\hline & Mean & -0.808844 & -0.808844 & -0.808440 & -0.808775 & -0.807086 & 0.767928 \\
\hline & Std & $2.22 E-16$ & $3.25 E-11$ & $2.95 E-03$ & $6.84 E-04$ & $5.63 E-03$ & $1.65 E+00$ \\
\hline & $t$-test & - & - & 0 & 0 & 1 & 1 \\
\hline & $W$-test & - & - & 0 & 0 & 1 & 1 \\
\hline \multirow{5}{*}{ F11 } & Best & -0.974565 & -0.974565 & -0.974565 & -0.974565 & -0.974565 & -0.974565 \\
\hline & Mean & -0.974565 & -0.974565 & -0.974565 & -0.974255 & -0.974565 & -0.961576 \\
\hline & Std & $1.11 E-16$ & $1.56 E-15$ & $1.11 E-16$ & $2.12 E-03$ & $1.11 E-16$ & $1.27 E-02$ \\
\hline & $t$-test & - & - & 0 & 0 & 0 & 1 \\
\hline & $W$-test & - & - & 0 & 1 & 0 & 1 \\
\hline
\end{tabular}


TABLE 1: Continued.

\begin{tabular}{|c|c|c|c|c|c|c|c|}
\hline Fun & Metric & AHcHLO & HcHLO & ASHLO & IAHLO & SFPSO & ALPSO \\
\hline \multirow{5}{*}{ F12 } & Best & -1.000000 & -0.999892 & -1.000000 & -1.000000 & -1.000000 & -1.000000 \\
\hline & Mean & -1.000000 & -0.999821 & -1.000000 & -1.000000 & --1.000000 & -0.999999 \\
\hline & Std & $0.00 E+00$ & $9.54 E-06$ & $4.01 E-08$ & $1.40 E-08$ & $0.00 E+00$ & $3.22 E-06$ \\
\hline & $t$-test & - & - & 1 & 0 & 0 & 1 \\
\hline & $W$-test & - & - & 1 & 0 & 0 & 1 \\
\hline \multirow{5}{*}{ F13 } & Best & 5850.383000 & 5850.438514 & 5850.961000 & 5850.522000 & 6090.693000 & 5903.295000 \\
\hline & Mean & 5974.989830 & 5908.944814 & 6010.429390 & 5980.964330 & 6104.016650 & 6347.670080 \\
\hline & Std & $1.08 E+02$ & $1.01 E+02$ & $1.11 E+02$ & $1.21 E+02$ & $4.94 E+01$ & $2.92 E+02$ \\
\hline & $t$-test & - & - & 1 & 0 & 1 & 1 \\
\hline & $W$-test & - & - & 1 & 0 & 1 & 1 \\
\hline \multirow{5}{*}{ F14 } & Best & -75.134170 & -75.134137 & -75.132390 & -75.133670 & -75.133990 & -75.131530 \\
\hline & Mean & -75.134170 & -75.134137 & -74.880659 & -74.919266 & -74.958547 & -72.689203 \\
\hline & Std & $2.84 E-14$ & $1.11 E-07$ & $8.38 E-02$ & $1.11 E-01$ & $1.37 E-01$ & $2.65 E+00$ \\
\hline & $t$-test & - & - & 1 & 1 & 1 & 1 \\
\hline & $W$-test & - & - & 1 & 1 & 1 & 1 \\
\hline
\end{tabular}

The best numbers are given in bold, and the lower values of results indicate better optimization ability.

TABLe 2: The summary results of the $t$-test and $W$-test on the benchmark functions.

\begin{tabular}{|c|c|c|c|c|c|}
\hline & HcHLO & ASHLO & IAHLO & SFPSO & ALPSO \\
\hline \multicolumn{6}{|c|}{$t$-test } \\
\hline 1 & - & 8 & 6 & 8 & 8 \\
\hline 0 & - & 6 & 8 & 6 & 6 \\
\hline-1 & - & 0 & 0 & 0 & 0 \\
\hline \multicolumn{6}{|c|}{$W$-test } \\
\hline 1 & - & 9 & 8 & 8 & 9 \\
\hline 0 & - & 5 & 6 & 6 & 5 \\
\hline-1 & - & 0 & 0 & 0 & 0 \\
\hline
\end{tabular}

Three indicators, i.e., the best value (Best), the mean best value (Mean), and the standard deviation (Std), are used to evaluate the performance of AHcHLO. The numerical results of all the cases are shown in Table 1, where the best numbers are in bold, and the lower values of results indicate better optimization ability. The paired Student's $t$-test $(t$ test) and the Wilcoxon signed-rank test ( $W$-test) results are also listed in Table 1, in which " 0 " indicates that AHcHLO is comparable to the other algorithms, " 1 " and " -1 " mean that the performance of AHcHLO is significantly better and worse than the other algorithms in the $95 \%$ confidence interval, respectively. Note that the $t$-test assumes Gaussian distribution while the $W$-test does not. Therefore, the $t$-test is more reliable when the Gaussian distribution assumption is met while the $W$-test would be more powerful when this assumption is violated [49]. For convenience, the results of the $t$-test and $W$-test are summarized in Table 2 .

Table 1 shows that the proposed AHcHLO obtains the best numerical results on 11 out of 14 functions. Besides, the summary results of the $t$-test in Table 2 indicate that the proposed AHcHLO surpasses ASHLO, IAHLO, SFPSO, and ALPSO on $8,6,8$, and 8 out of 14 functions. Moreover, the $W$-test results prove that the proposed AHcHLO significantly outperforms these compared algorithms on $9,8,8$, and 9 out of 14 functions, respectively. Note that $\mathrm{HcHLO}$ has obtained the best-known overall results on this set of hybridcoded benchmark functions before this work [27], in which the HcHLO significantly surpasses MA-MDE', MDE'-IHS,
$\mathrm{MDE}^{\prime}-\mathrm{HJ}$, and $\mathrm{MDE}^{\prime}-\mathrm{IHS}-\mathrm{HJ}$ on all the functions. Therefore, AHcHLO possesses the best-known overall results so far on this set of hybrid-coded benchmark functions because the developed adaptive strategy can further enhance the optimization search ability of AHcHLO. It is fair to claim that AHcHLO is a very promising optimization tool for hybrid-coded problems in scientific research and engineering applications and has an advantage over the studied segmentation model.

4.2. Segmentation Simulation for Furnace Flame. The proposed NACMM was adopted to identify the flame images of different combustion states, which was compared with the three recent methods, i.e., statistical color model (SCM) [11], ICA K-medoids-based color model (ICA-KCM) [17], and new conversion-based target-oriented color space model (NCTCSM) [21]. The comparison results of flame segmentation of original images I-XV are displayed in Figure 8, in which Figure 8(a) shows the original flame images I-XV and Figures 8(b)-8(e) indicate the segmentation effects of SCM, ICA-KCM, NCTCSM, and NACMM, respectively. Besides, for quantitative comparison, two evaluation metrics, i.e., detection accuracy (DA) and error rate (ER), were adopted to objectively evaluate the performance of all the methods. DA/ER is the proportion of the number of correctly/wrongly classified pixels (flame and nonflame) to the total number of pixels, respectively. Higher values of DA and 

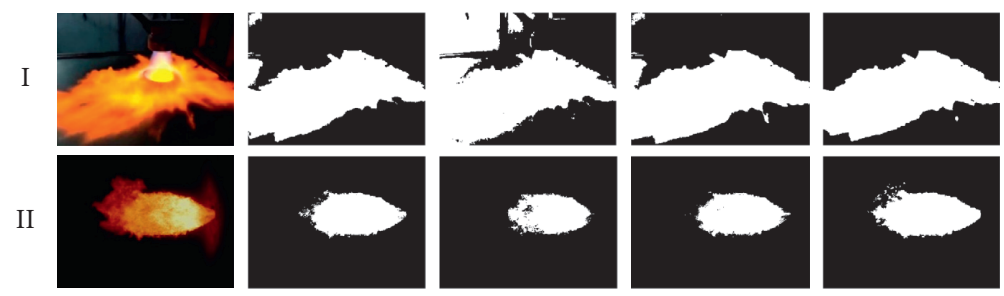

II
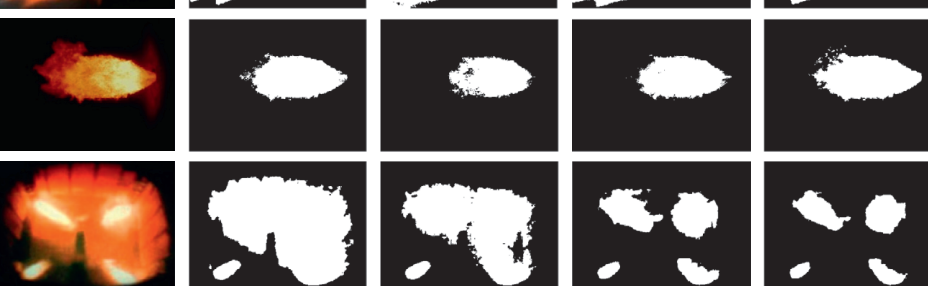

III
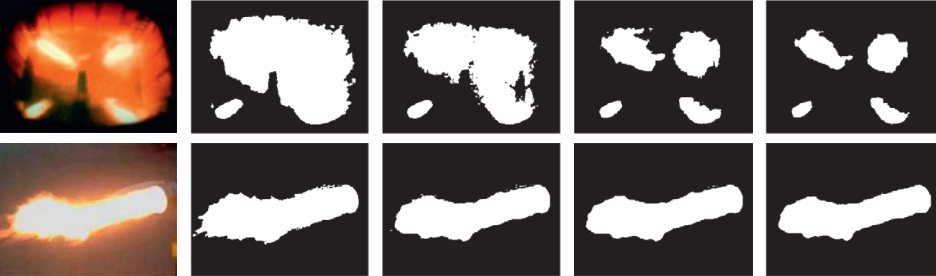

IV
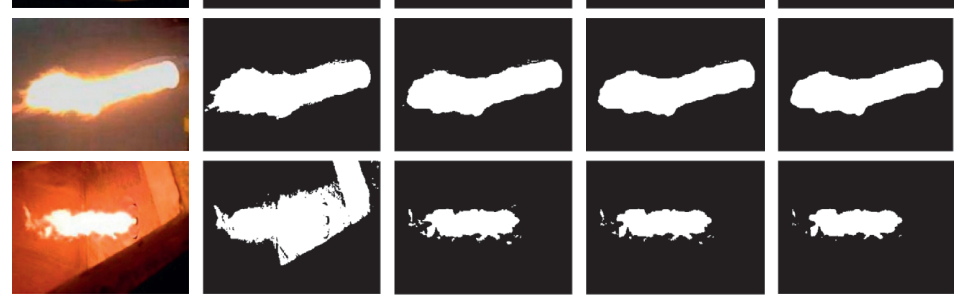

V
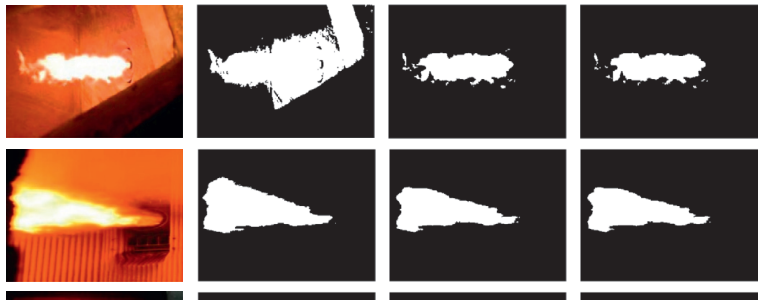

thenters
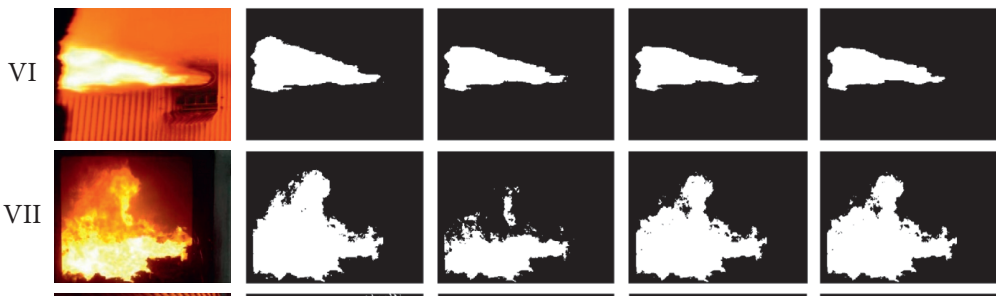

VII
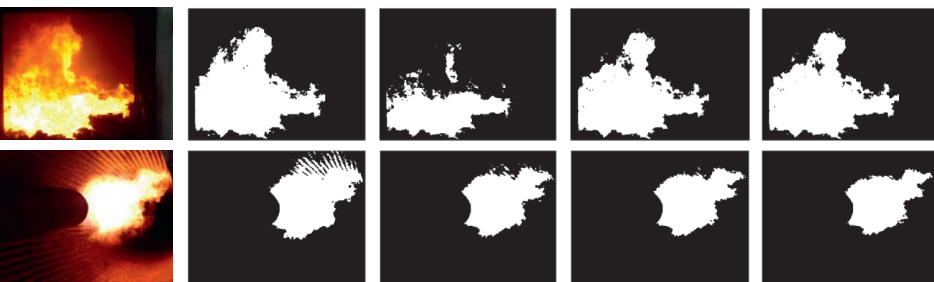

VIII

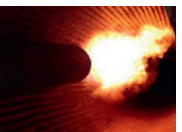

IX
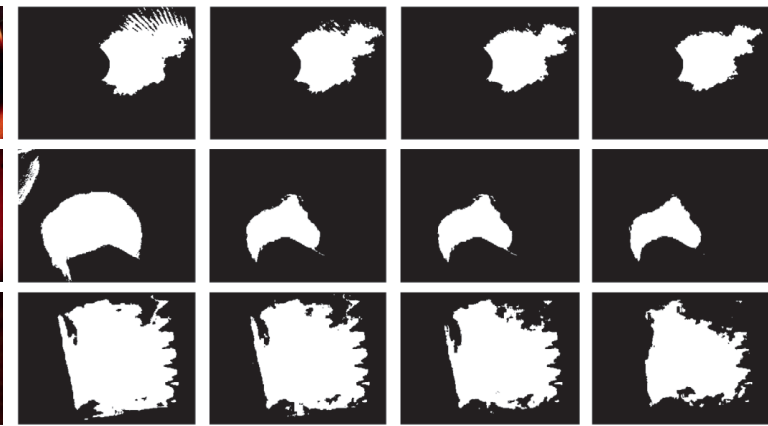

X
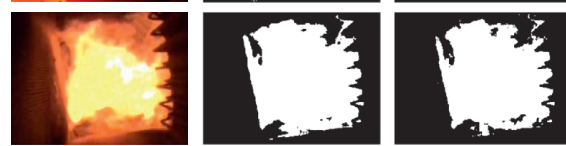

XI
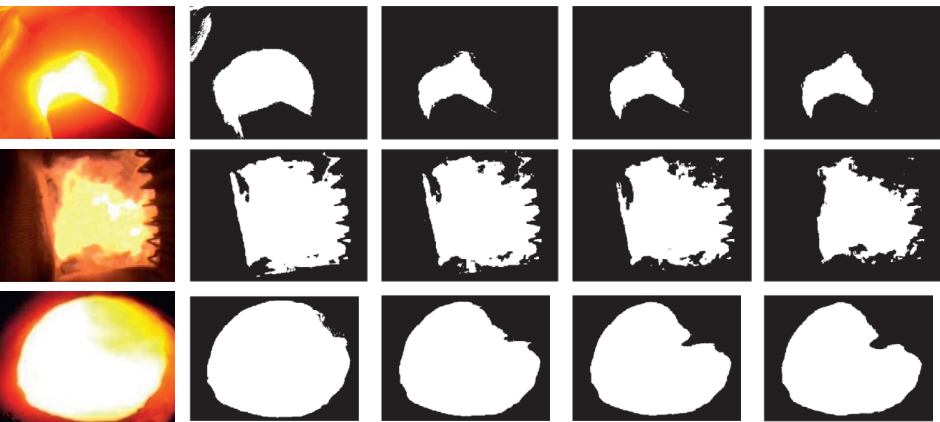

XII
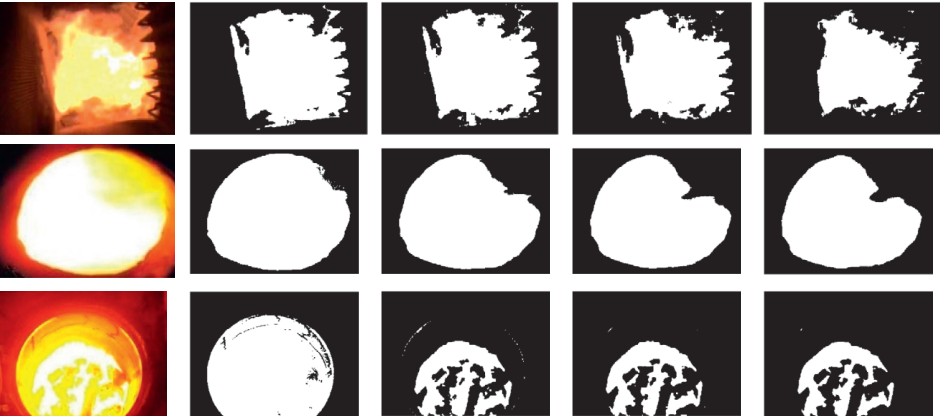

XIII
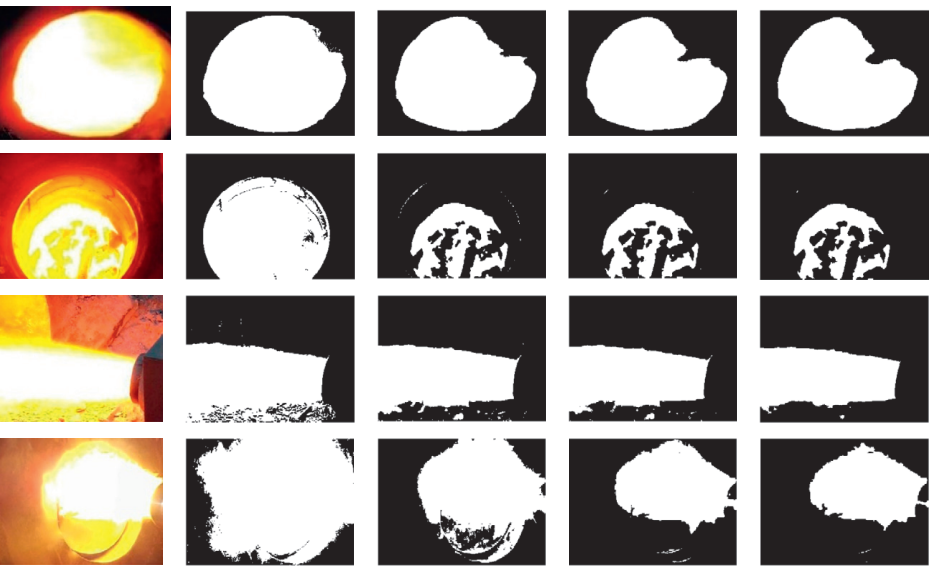

XIV

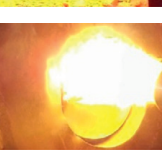

XV

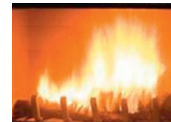

(a)

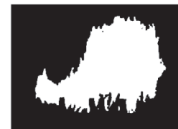

(b)

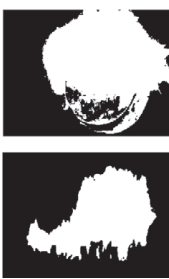

(c)

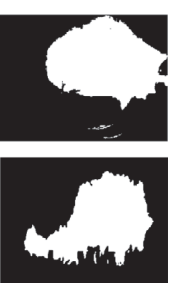

(d)
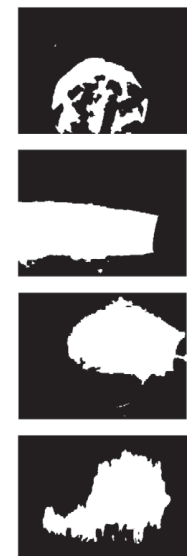

(e)

FIGURE 8: The comparison results of flame segmentation of original images I-XV: (a) original images I-XV; (b) results from SCM; (c) results from ICA-KCM; (d) results from NCTCSM; (e) results from proposed NACMM. 
TABLE 3: The comparison metrics of flame segmentation of original images I-XV.

\begin{tabular}{|c|c|c|c|c|c|}
\hline \multirow{2}{*}{ Original image } & \multirow{2}{*}{ Method } & \multicolumn{4}{|c|}{ Evaluation metrics } \\
\hline & & False pixels & Correct pixels & ER (\%) & DA (\%) \\
\hline \multirow{4}{*}{ Original image I } & SCM & 9325 & 135875 & 6.42 & 93.58 \\
\hline & ICA-KCM & 30541 & 114659 & 21.03 & 78.97 \\
\hline & NCTCSM & 6316 & 138884 & 4.35 & 95.65 \\
\hline & Proposed NACMM & 4137 & 141063 & 2.85 & 97.15 \\
\hline \multirow{4}{*}{ Original image II } & SCM & 7800 & 112200 & 6.50 & 93.50 \\
\hline & ICA-KCM & 11021 & 108979 & 9.18 & 90.82 \\
\hline & NCTCSM & 10750 & 109250 & 8.96 & 91.04 \\
\hline & Proposed NACMM & 6004 & 113996 & 5.00 & 95.00 \\
\hline \multirow{4}{*}{ Original image III } & SCM & 37648 & 59552 & 38.73 & 61.27 \\
\hline & ICA-KCM & 26367 & 70833 & 27.13 & 72.87 \\
\hline & NCTCSM & 9255 & 87945 & 9.52 & 90.48 \\
\hline & Proposed NACMM & 5889 & 91311 & 6.06 & 93.94 \\
\hline \multirow{4}{*}{ Original image IV } & SCM & 2821 & 73979 & 3.67 & 96.33 \\
\hline & ICA-KCM & 1721 & 75079 & 2.24 & 97.76 \\
\hline & NCTCSM & 2319 & 74481 & 3.02 & 96.98 \\
\hline & Proposed NACMM & 1545 & 75255 & 2.01 & 97.99 \\
\hline \multirow{4}{*}{ Original image $\mathrm{V}$} & SCM & 28336 & 103964 & 21.42 & 78.58 \\
\hline & ICA-KCM & 2686 & 129614 & 2.03 & 97.97 \\
\hline & NCTCSM & 2538 & 129762 & 1.92 & 98.08 \\
\hline & Proposed NACMM & 2194 & 130106 & 1.66 & 98.34 \\
\hline \multirow{4}{*}{ Original image VI } & SCM & 7050 & 112950 & 5.88 & 94.13 \\
\hline & ICA-KCM & 2667 & 117333 & 2.22 & 97.78 \\
\hline & NCTCSM & 2495 & 117505 & 2.08 & 97.92 \\
\hline & Proposed NACMM & 1430 & 118570 & 1.19 & 98.81 \\
\hline \multirow{4}{*}{ Original image VII } & SCM & 4156 & 115844 & 3.46 & 96.54 \\
\hline & ICA-KCM & 14387 & 105613 & 11.99 & 88.01 \\
\hline & NCTCSM & 1957 & 118043 & 1.63 & 98.37 \\
\hline & Proposed NACMM & 1884 & 118116 & 1.57 & 98.43 \\
\hline \multirow{4}{*}{ Original image VIII } & SCM & 7371 & 79329 & 8.50 & 91.50 \\
\hline & ICA-KCM & 4135 & 82565 & 4.77 & 95.23 \\
\hline & NCTCSM & 3569 & 83131 & 4.12 & 95.88 \\
\hline & Proposed NACMM & 2488 & 84212 & 2.87 & 97.13 \\
\hline \multirow{4}{*}{ Original image IX } & SCM & 23723 & 134977 & 14.95 & 85.05 \\
\hline & ICA-KCM & 2369 & 156331 & 1.49 & 98.51 \\
\hline & NCTCSM & 2401 & 156299 & 1.51 & 98.49 \\
\hline & Proposed NACMM & 737 & 157963 & 0.46 & 99.54 \\
\hline \multirow{4}{*}{ Original image $\mathrm{X}$} & SCM & 32349 & 140451 & 18.72 & 81.28 \\
\hline & ICA-KCM & 28058 & 144742 & 16.24 & 83.76 \\
\hline & NCTCSM & 21830 & 150970 & 12.63 & 87.37 \\
\hline & Proposed NACMM & 9838 & 162962 & 5.69 & 94.31 \\
\hline \multirow{4}{*}{ Original image XI } & SCM & 10153 & 66647 & 13.22 & 86.78 \\
\hline & ICA-KCM & 5626 & 71174 & 7.33 & 92.67 \\
\hline & NCTCSM & 3887 & 72913 & 5.06 & 94.94 \\
\hline & Proposed NACMM & 2696 & 74104 & 3.51 & 96.49 \\
\hline \multirow{4}{*}{ Original image XII } & SCM & 27593 & 49207 & 35.93 & 64.07 \\
\hline & ICA-KCM & 3579 & 73221 & 4.66 & 95.34 \\
\hline & NCTCSM & 2356 & 74444 & 3.07 & 96.93 \\
\hline & Proposed NACMM & 1630 & 75170 & 2.12 & 97.88 \\
\hline \multirow{4}{*}{ Original image XIII } & SCM & 11965 & 74735 & 13.80 & 86.20 \\
\hline & ICA-KCM & 5167 & 81533 & 5.96 & 94.04 \\
\hline & NCTCSM & 4446 & 82254 & 5.13 & 94.87 \\
\hline & Proposed NACMM & 2551 & 84149 & 2.94 & 97.06 \\
\hline \multirow{4}{*}{ Original image XIV } & SCM & 59654 & 60346 & 49.71 & 50.29 \\
\hline & ICA-KCM & 25457 & 94543 & 21.21 & 78.79 \\
\hline & NCTCSM & 10266 & 109734 & 8.56 & 91.45 \\
\hline & Proposed NACMM & 5325 & 114675 & 4.44 & 95.56 \\
\hline
\end{tabular}


TABle 3: Continued.

\begin{tabular}{|c|c|c|c|c|c|}
\hline \multirow{2}{*}{ Original image } & \multirow{2}{*}{ Method } & \multicolumn{4}{|c|}{ Evaluation metrics } \\
\hline & & False pixels & Correct pixels & ER (\%) & DA (\%) \\
\hline \multirow{4}{*}{ Original image XV } & SCM & 8268 & 59232 & 12.25 & 87.75 \\
\hline & ICA-KCM & 7025 & 60475 & 10.41 & 89.59 \\
\hline & NCTCSM & 6809 & 60691 & 10.09 & 89.91 \\
\hline & Proposed NACMM & 5554 & 61946 & 8.23 & 91.77 \\
\hline \multirow{4}{*}{ All original images } & SCM & 278212 & 1379288 & 16.79 & 83.21 \\
\hline & ICA-KCM & 170806 & 1486694 & 10.31 & 89.69 \\
\hline & NCTCSM & 91194 & 1566306 & 5.50 & 94.50 \\
\hline & Proposed NACMM & 53902 & 1603598 & 3.25 & 96.75 \\
\hline
\end{tabular}

The best results are marked in bold. Higher values of DA and lower values of ER indicate better accuracies.

lower values of ER indicate better accuracies. The comparison metrics of flame segmentation of original images I-XV are listed in Table 3, where the best results have been marked with boldface.

Figure 8 and Table 3 clearly show that the proposed method can effectively improve the detection accuracy and the segmentation effect of combustion flame. The characteristics of the NACMM as well as the other three recent methods can be concluded as follows:

(1) From Figure 8(b), the segmentation effects of original images I, II, III, V, VIII, IX, X, XI, XII, XIII, XIV by using SCM are not ideal, which cannot effectively distinguish flame pixels and nonflame pixels. In particular, for the original images III, V, IX, X, XI, XII, XIII, XIV, the segmentation effect is poor and the numerical values of ER are large. Table 3 shows that the DAs of original images I-XV by using SCM are quite different and the numerical values of DA are $93.58 \%, 93.50 \%, 61.27 \%, 96.33 \%, 78.58 \%, 94.13 \%$, $96.54 \%, 91.50 \%, 85.05 \%, 81.28 \%, 86.78 \%, 64.07 \%$, $86.2 \%, 50.29 \%$, and $87.75 \%$, respectively, which points out that SCM cannot segment the flame image of different combustion status.

(2) Figure 8(c) shows that the ICA-KCM has the same disadvantages of SCM, which cannot effectively segment the flame image of different combustion status, in which the segmentation effects of original images I, II, III, VII, X, XI, XIV by using ICA-KCM are also not ideal.

(3) Figure 8(d) indicates that the segmentation effect and the detection accuracy of NCTCSM are better than those of SCM and ICA-KCM because it uses the PSO algorithm to optimize the color space model with nine variables, which can segment the flame pixels of different combustion states. However, this recognition model is relatively complicated; it is difficult to obtain a high-precision recognition model, which causes the values of DA of NCTCSM to be lower than those of NACMM.

(4) Figure 8(e) points out that the proposed NACMM can effectively identify the flame image of different combustion statuses, which obtains the best segmentation effect and the highest detection accuracy of combustion flame. Specifically, the DA of original images I-XV are 97.15\%, 95.00\%, 93.94\%, 97.99\%, 98.34\%, 98.81\%, 98.43\%, 97.13\%, 99.54\%, 94.31\%, $96.49 \%, 97.88 \%, 97.06 \%, 95.56 \%$, and $91.77 \%$, respectively, which are better than those of SCM, ICAKCM, and NCTCSM. The numerical values of DA for fifteen combustion flame images I-XV are greater than $90 \%$, which proves that the proposed NACMM has good robustness. Besides, the results of the average values of ER and DA also demonstrate the superiority of NACMM, which obtains the best numerical results; that is, the values of ER and DA are $3.25 \%$ and $96.75 \%$, respectively.

In conclusion, the segmentation effects of both SCM and ICA-KCM are not ideal because they do not have the objective function to adapt to different combustion states, and it is difficult for the NCTCSM to obtain a high-precision segmentation model because the optimized model parameters are complicated. Compared with SCM, ICA-KCM, and NCTCSM, the proposed NACMM can segment the flame image of different combustion statuses more effectively and accurately, which can obtain an ideal adaptive segmentation model for adapting to different combustion states.

\section{Conclusions and Future Work}

Designing and developing high detection accuracy technology for flame segmentation are crucial, which can effectively help operators adjust combustion strategies for improving combustion utilization. However, the combustion states, as well as the RGB components of combustion flame, inside the industrial furnace change according to the production needs, which is not considered in the previous works and further challenges the optimal set of model parameters. Therefore, a novel segmentation method for furnace flame using adaptive color model and hybrid-coded HLO is proposed to segment the flame pixels more accurately and effectively, in which the NACMM is designed for adapting to the flame image of different combustion states. As the objective function of NACMM is a hybrid-coding problem, the AHcHLO is developed to solve the optimized parameters of NACMM. Regarding this proposed NACMM, two objective functions are adopted as the evaluation index to evaluate the segmentation accuracy and reduce the structural risk. Firstly, AHcHLO is applied to solve the benchmark functions for evaluating its optimization ability, 
and the numerical results show that AHcHLO possesses the best-known overall results so far on these benchmark functions, which further ensures the parameter optimization of NACMM for guaranteeing the best effect. Then, the segmentation simulation demonstrates that the proposed NACMM outperforms state-of-the-art flame segmentation approaches in detection accuracy and segmentation effect for different combustion states.

The color model is extremely important for the segmentation effect of flame image, and the hue and saturation can also influence the segmentation effect of flame image. Therefore, the following research will focus on studying the advanced yet complicated color model with mixed variables to further improve the detection accuracy of combustion flame. However, considering that this color model contains more variables, it needs a more powerful HLO algorithm to obtain the best color model, which will be challenging for future work.

\section{Data Availability}

The data used to support the findings of this study are available from the corresponding author upon request.

\section{Conflicts of Interest}

The authors declare that they have no conflicts of interest.

\section{Acknowledgments}

This work was supported by the National Natural Science Foundation of China under Grant nos. 61833011, 61633016, and 92067105; Key Project of Science and Technology Commission of Shanghai Municipality under Grant nos. 16010500300, 19510750300, and 19500712300; and 111 Project under Grant no. D18003.

\section{References}

[1] M. Öge, D. Özkan, M. B. Celik, M. Sabri Gok, and A. Cahit Karaoglanli, "An overview of utilization of blast furnace and steelmaking slag in various applications," Materials Today: Proceedings, vol. 11, pp. 516-525, 2019.

[2] F. Alobaid, J.-P. Busch, A. Stroh, J. Ströhle, and B. Epple, "Experimental measurements for torrefied biomass Cocombustion in a 1 MWth pulverized coal-fired furnace," Journal of the Energy Institute, vol. 93, no. 3, pp. 833-846, 2020.

[3] C. Gao, M. Shen, X. Liu, L. Wang, and M. Chu, "End-point static control of basic oxygen furnace (BOF) steelmaking based on wavelet transform weighted twin support vector regression," Complexity, vol. 2019, Article ID 7408725, 16 pages, 2019.

[4] Z. Wang, Y. Bai, J. Xie et al., "Fixed-time sliding-mode faulttolerant control of waste heat power generator systems," Complexity, vol. 2018, Article ID 3580628, 8 pages, 2018.

[5] H. Mikulcic, E. von Berg, X. Wang et al., "Using an advanced numerical technique for improving pulverized coal combustion inside an industrial furnace," Chemical Engineering Transactions, vol. 61, pp. 235-240, 2017.

[6] J. Chang, J. Wang, Z. Wang, S. Shan, and C. Liu, "Analysis of converter combustion flame spectrum big data sets based on
HHT," Complexity, vol. 2018, Article ID 8682725, 11 pages, 2018.

[7] Z. Ouyang, W. Liu, C. Man, J. Zhu, and J. Liu, "Experimental study on combustion, flame and NOX emission of pulverized coal preheated by a preheating burner," Fuel Processing Technology, vol. 179, pp. 197-202, 2018.

[8] J. Shen, M. Yang, B. Zou, N. Wan, and Y. Liao, "Outlier detection of air temperature series data using probabilistic finite state automata-based algorithm," Complexity, vol. 17, no. 5, pp. 48-57, 2012.

[9] A. E. Çetin, K. Dimitropoulos, B. Gouverneur et al., "Video fire detection - Review," Digital Signal Processing, vol. 23, no. 6, pp. 1827-1843, 2013.

[10] T. Wang, Q. Liu, H. Chen et al., "Temperature measurement method of flame images based on BP neural network," China Measurement Technology, vol. 2, 2005.

[11] T. Celik, H. Demirel, H. Ozkaramanli, and M. Uyguroglu, "Fire detection using statistical color model in video sequences," Journal of Visual Communication and Image Representation, vol. 18, no. 2, pp. 176-185, 2007.

[12] R. Zhang, Y. Cheng, Y. Li, D. Zhou, and S. Cheng, "Imagebased flame detection and combustion analysis for blast furnace raceway," IEEE Transactions on Instrumentation and Measurement, vol. 68, no. 4, pp. 1120-1131, 2019.

[13] Y. Chen, W. Xu, J. Zuo et al., "The fire recognition algorithm using dynamic feature fusion and IV-SVM classifier," Cluster Computing, vol. 22, no. 3, pp. 7665-7675, 2019.

[14] Y. Wang, L. Dang, and J. Ren, "Forest fire image recognition based on convolutional neural network," Journal of Algorithms \& Computational Technology, vol. 13, 2019.

[15] T. Qiu, M. Liu, G. Zhou, L. Wang, and K. Gao, "An unsupervised classification method for flame image of pulverized coal combustion based on convolutional auto-encoder and hidden Markov model," Energies, vol. 12, no. 13, p. 2585, 2019.

[16] P. G. Zhang, M. R. Fei, L. Wang et al., "Identification method for furnace flame based on adaptive color model," Social Science Information, vol. 48, pp. 1-15, 2018, in Chinese.

[17] M. Hashemzadeh and A. Zademehdi, "Fire detection for video surveillance applications using ICA K-medoids-based color model and efficient spatio-temporal visual features," Expert Systems with Applications, vol. 130, pp. 60-78, 2019.

[18] S. Ye, Z. Bai, H. Chen, R. Bohush, and S. Ablameyko, "An effective algorithm to detect both smoke and flame using color and wavelet analysis," Pattern Recognition and Image Analysis, vol. 27, no. 1, pp. 131-138, 2017.

[19] X. Bai, G. Lu, M. M. Hossain, Y. Yan, and S. Liu, "Multimode monitoring of oxy-gas combustion through flame imaging, principal component analysis, and kernel support vector machine," Combustion Science and Technology, vol. 189, no. 5, pp. 776-792, 2017.

[20] X.-F. Han, J. S. Jin, M.-J. Wang, W. Jiang, L. Gao, and L.-P. Xiao, "Video fire detection based on Gaussian Mixture Model and multi-color features," Signal, Image and Video Processing, vol. 11, no. 8, pp. 1419-1425, 2017.

[21] A. Khatami, S. Mirghasemi, A. Khosravi, C. P. Lim, and S. Nahavandi, "A new PSO-based approach to fire flame detection using K-Medoids clustering," Expert Systems with Applications, vol. 68, pp. 69-80, 2017.

[22] H. V. H. Ayala, F. M. d. Santos, V. C. Mariani, and L. d. S. Coelho, "Image thresholding segmentation based on a novel beta differential evolution approach," Expert Systems with Applications, vol. 42, no. 4, pp. 2136-2142, 2015.

[23] O. Banimelhem, M. Mowafi, and O. Alzoubi, "Multilevel thresholding image segmentation using memetic algorithm," 
in Proceedings of the 2015 6th International Conference on Information and Communication Systems (ICICS), pp. 119123, Amman, Jordan, 2015.

[24] A. Khan, Z. ur Rehman, M. A. Jaffar et al., "Color image segmentation using genetic algorithm with aggregation-based clustering validity index (CVI)," Signal, Image and Video Processing, vol. 13, no. 5, pp. 833-841, 2019.

[25] M. S. Imbabi, C. Carrigan, and S. McKenna, "Trends and developments in green cement and concrete technology," International Journal of Sustainable Built Environment, vol. 1, no. 2, pp. 194-216, 2012.

[26] A. K. Bhandari and I. V. Kumar, "A context sensitive energy thresholding based 3D Otsu function for image segmentation using human learning optimization," Applied Soft Computing, vol. 82, Article ID 105570, 2019.

[27] L. Wang, J. Pei, M. I. Menhas, J. Pi, M. Fei, and P. M. Pardalos, "A hybrid-coded human learning optimization for mixedvariable optimization problems," Knowledge-Based Systems, vol. 127, pp. 114-125, 2017.

[28] L. Wang, H. Ni, R. Yang, M. Fei, and W. Ye, "A simple human learning optimization algorithm," in Communications in Computer and Information Science, pp. 56-65, Springer, Berlin, Germany, 2014.

[29] L. Wang, R. Yang, H. Ni, W. Ye, M. Fei, and P. M. Pardalos, “A human learning optimization algorithm and its application to multi-dimensional knapsack problems," Applied Soft Computing, vol. 34, pp. 736-743, 2015.

[30] L. Wang, J. Pei, Y. Wen, J. Pi, M. Fei, and P. M. Pardalos, "An improved adaptive human learning algorithm for engineering optimization," Applied Soft Computing, vol. 71, pp. 894-904, 2018.

[31] J. Cao, Z. Yan, X. Xu, G. He, and S. Huang, "Optimal power flow calculation in AC/DC hybrid power system based on adaptive simplified human learning optimization algorithm," Journal of Modern Power Systems and Clean Energy, vol. 4, no. 4, pp. 690-701, 2016.

[32] R. Alguliyev, R. Aliguliyev, and N. Isazade, "A sentence selection model and HLO algorithm for extractive text summarization," in Proceedings of the 2016 IEEE 10th International Conference on Application of Information and Communication Technologies (AICT), pp. 1-4, Baku, Azerbaijan, 2016.

[33] R. Yang, J. He, M. Xu, H. Ni, P. Jones, and N. Samatova, “An intelligent and hybrid weighted fuzzy time series model based on empirical mode decomposition for financial markets forecasting," in Advances in Data Mining. Applications and Theoretical Aspectspp. 104-118, New York, NY, USA, 2018.

[34] W. Fan, J. Pei, X. Liu, P. M. Pardalos, and M. Kong, "Serialbatching group scheduling with release times and the combined effects of deterioration and truncated job-dependent learning," Journal of Global Optimization, vol. 71, no. 1, pp. 147-163, 2018.

[35] Y. Wen, L. Wang, W. Peng, M. I. Menhas, and L. Qian, "Application of intelligent virtual reference feedback tuning to temperature control in a heat exchanger," in Intelligent Computing and Internet of Things, pp. 311-320, Springer, Singapore, 2018.

[36] L. Blier, P. Wolinski, and Y. Ollivier, "Learning with random learning rates," in Proceedings of the Joint European Conference on Machine Learning and Knowledge Discovery in Databases, pp. 449-464, Würzburg, Germany, 2019.

[37] A. Mesoudi, L. Chang, S. R. X. Dall, and A. Thornton, "The evolution of individual and cultural variation in social learning," Trends in Ecology \& Evolution, vol. 31, no. 3, pp. 215-225, 2016.

[38] H. Over and C. McCall, "Becoming us and them: social learning and intergroup bias," Social and Personality Psychology Compass, vol. 12, no. 4, Article ID e12384, 2018.

[39] A. T. Qamar, R. J. Cotton, R. G. George et al., "Trial-to-trial, uncertainty-based adjustment of decision boundaries in visual categorization," Proceedings of The National Academy of Sciences, vol. 110, no. 50, pp. 20332-20337, 2013.

[40] B. J. Zimmerman and M. Martinez-Pons, "Construct validation of a strategy model of student self-regulated learning," Journal of Educational Psychology, vol. 80, no. 3, pp. 284-290, 1988.

[41] K. H. Hoffmann, A. Franz, and P. Salamon, "Structure of best possible strategies for finding ground states," Physical Reviewe Statistical Nonlinear \& Soft Matter Physics, vol. 66, no. 42, Article ID 046706, 2002.

[42] H. Scarbrough, J. Swan, S. Laurent, M. Bresnen, L. Edelman, and S. Newell, "Project-based learning and the role of learning boundaries," Organization Studies, vol. 25, no. 9, pp. 1579-1600, 2004.

[43] L. Wang, H. Ni, R. Yang, P. M. Pardalos, X. Du, and M. Fei, "An adaptive simplified human learning optimization algorithm," Information Sciences, vol. 320, pp. 126-139, 2015.

[44] A. M. Panditrao and P. P. Rege, "Temperature estimation of visible heat sources by digital photography and image processing," IEEE Transactions on Instrumentation and Measurement, vol. 59, no. 5, pp. 1167-1174, 2010.

[45] Y. Xu, S. Li, Y. Yuan, and Q. Yao, "Measurement on the surface temperature of dispersed chars in a flat-flame burner using modified RGB pyrometry," Energy \& Fuels, vol. 31, no. 3, pp. 2228-2235, 2017.

[46] S. L. Gupta, A. S. Baghel, and A. Iqbal, "Big data classification using scale-free binary particle swarm optimization," in Harmony Search and Nature Inspired Optimization Algorithms, pp. 1177-1187, Springer, Singapore, 2019.

[47] F. Wang, H. Zhang, K. Li, Z. Lin, J. Yang, and X.-L. Shen, “A hybrid particle swarm optimization algorithm using adaptive learning strategy," Information Sciences, vol. 436-437, pp. 162-177, 2018.

[48] H. Yi, Q. Duan, and T. W. Liao, "Three improved hybrid metaheuristic algorithms for engineering design optimization," Applied Soft Computing, vol. 13, no. 5, pp. 2433-2444, 2013.

[49] L. Wang, L. An, J. Pi et al., "A diverse human learning optimization algorithm," Journal of Global Optimization, vol. 67, no. 1-2, pp. 283-323, 2017. 